\title{
Contexto paleoambiental del yacimiento Neolítico de Los Cascajos (Navarra, España): quimioestratigrafía de la secuencia sedimentaria holocena del río Odrón
}

\author{
Eneko Iriarte, Miren del Val, Iñigo García-Martínez de Lagrán, Cristina Tejedor-Rodríguez,
} Jesús García-Gazólaz, Jesús Sesma-Sesma, Manuel A. Rojo-Guerra

\begin{abstract}
Eneko Iriarte
eiriarte@ubu.es

Laboratorio de Evolución Humana, Departamento de Historia, Geografia y Comunicación, Universidad de Burgos, Plaza Misael Bañuelos, Edificio de I+D+i, 09001 Burgos, España.
\end{abstract}

\section{Miren del Val}

Departmento de Mineralogía y Petrología, Facultad de Ciencias y Tecnología, UPV/EHU Universidad del País Vasco, Barrio Sarriena s/n, 48940 Leioa, España.

Centro Nacional de Investigación sobre la Evolución Humana (CENIEH), Paseo Sierra de Atapuerca, 3, 09002 Burgos, España.

\section{Iñigo García-Martínez de Lagrán Manuel A. Rojo-Guerra \\ Departamenteo de Prehistoria y Arqueología, Universidad de Valladolid, Plaza del Campus, 47011 Valladolid, España.}

\section{Gristina Tejedor-Rodríguez}

Instituto de Ciencias del Patrimonio-Consejo Superior de Investigaciones Científicas (INCIPIT-CSIC), Avda. de Vigo s/n, 15705

Santiago de Compostela, España.

\section{Jesús García-Gazólaz}

Jesús Sesma-Sesma

Dirección General de Cultura-Institución Príncipe de Viana, Gobierno de Navarra, C/ Navarrería 39, 31001 Pamplona, España.

BOL. SOC. GEOL. MEX. 2019

VOL. 71 NO. 2

P. $261-273$

http://dx.doi.org/10.18268/BSGM2019v7 ln2a3

\section{RESUMEN}

El conocimiento de los cambios climáticos y ambientales del inicio del Holoceno y su incidencia en las poblaciones y culturas humanas es clave para la comprensión de la evolución tanto pretérita como futura de éstas. En este sentido, el estudio mediante zanjas y sondeos del registro arqueosedimentario holoceno en el área del yacimiento neolítico de Los Cascajos (Los Arcos, Navarra), aporta una valiosa información no sólo para el establecimiento de la evolución ambiental de esta zona, sino también para el conocimiento de las actividades que los pobladores del yacimiento realizaron en los márgenes del río Odrón. En este trabajo se presentan los resultados del estudio estratigráfico, sedimentológico y geoquímico mediante XRF-CS de alta resolución del sondeo LAC1. Los datos obtenidos denotan una tendencia hacia la aridificación a lo largo del Holoceno, representada por un ciclo de agradación-incisión en la evolución fluvial del río Odrón. En el registro sedimentario fluvial se detectan también indicadores de actividad antrópica coetánea al asentamiento de Los Cascajos, lo que permite situar su ocupación en momentos hidrológicamente más húmedos que el actual, y evidenciar la posterior aridificación de esta zona del valle del Ebro a lo largo del Holoceno reciente (Megalayense).

Palabras clave: sedimentación fluvial, Holoceno, quimioestratigrafía, evolución paleoambiental, Neolítico, Los Cascajos.

\section{ABSTRACT}

The knowledge of climatic and environmental changes at the onset of the Holocene and its impact on human populations and cultures is key for the understanding of their both past and future evolution. In this sense, the study through trenching and cores of the Holocene archaeosedimentary record in the surroundings of the Los Cascajos Neolithic archaeological site (Los Arcos, Navarra), provides valuable information not only for the establishment of the environmental evolution of this area, but also for the knowledge of the activities that the inhabitants of the settlement developed in the Odrón riverbanks. In this work the results of the stratigraphic, sedimentological and geochemical study (XRFCS) of the LAC1 core are presented. The obtained data denote a trend towards longterm aridification throughout the Holocene, recorded by an aggradation-incision cycle in the fluvial evolution of the Odrón River. In the fluvial sedimentary record, anthropic activity proxies coetaneous to Los Cascajos settlement are also detected during a relatively more humid period than nowadays, this fact evidences the subsequent aridification of this area of the Ebro valley along the late Holocene (Meghalayan).

Keywords: fluvial sedimentation, Holocene, chemostratigraphy, palaeoenvironmental evolution, Neolithic, Los Cascajos. 


\section{Introducción}

El inicio del Holoceno se caracteriza por englobar numerosos cambios, desde climáticos y ambientales hasta de poblaciones y culturas humanas. La evolución tanto paleoambiental como la de los grupos humanos del inicio del Holoceno en el alto valle del Ebro es relativamente poco conocida en comparación al resto de la cuenca (Alday et al., 2018) y sin embargo, a partir de las investigaciones realizadas, ambas parecen estar íntimamente relacionadas (González-Sampériz et al., 2008, 2017; Aranbarri et al., 2014; García-Martínez de Lagrán et al., 2016). En este sentido, el descubrimiento y estudio del registro arqueosedimentario de inicios del Holoceno en el área del yacimiento de Los Cascajos (Los Arcos, Navarra) presentado en este artículo, aporta una valiosa información no sólo para el establecimiento de la evolución ambiental de esta zona, especialmente sensible a los cambios climáticos, sino también para el conocimiento de las actividades que los pobladores del yacimiento realizaron en los márgenes del río Odrón.

El yacimiento neolítico de Los Cascajos se localiza en el término municipal de Los Arcos (Navarra). La zona excavada se ubica sobre una terraza pleistocena del río Odrón a $6 \mathrm{~m}$ de altura sobre su cauce actual, y tanto el área del yacimiento como el valle del río, se desarrollan sobre una colina alargada compuesta por arcillas rojas, areniscas y yesos continentales cenozoicos del Mioceno (Aquitaniense). El poblado neolítico comprende una extensión cercana a las 80 ha y es uno de los asentamientos al aire libre más importantes de la Península Ibérica en la prehistoria reciente. La característica arqueológica más notable del poblado son las más de 600 estructuras negativas (hoyos, agujeros y zanjas interpretadas como silos, hogares, basureros, enterramientos, estructuras de combustión, etc.) que lo componen, y el foso perimetral que delimita el conjunto, englobando un importante registro arqueológico a lo largo de 4 fases de ocupación: Fase I (Neolítico Antiguo), Fase II (Neolítico Medio), Fase III (Neolítico Final-Calcolítico) y evidencias más esporádicas de una Fase IV (Bronce Final-Edad del Hierro) (García-Gazólaz y Sesma-Sesma, 2001, 2005; Peña-Chocarro et al., 2005). Sobre el nivel de grava subyacente no se ha podido constatar una secuencia estratigráfica, pues los apenas $45 \mathrm{~cm}$ de suelo están alterados por la erosión y revueltos por las labores agrícolas, que han profundizado hasta el nivel de gravas de la terraza.

Dado el carácter superficial del yacimiento, sin apenas estratigrafía conservada, durante septiembre del año 2014 se realizó una campaña de prospección y muestreo del registro arqueosedimentario que permitieran contextualizarlo paleoambientalmente.

Para ello se realizaron 2 sondeos y 2 excavaciones mecánicas (zanjas) en las márgenes del río Odrón (Figura 1). Tanto las dos zanjas (Figura 1A y 1B) como uno de los sondeos (LAC1) se realizaron dentro del área del yacimiento, a orillas del río Odrón. El sondeo LA1, sin embargo, se realizó 2.5 $\mathrm{km}$ aguas abajo de los anteriores, en una amplia depresión fácilmente inundable en el cauce del río Odrón (LA1 en Figura 1). Todos los sondeos y excavaciones permitieron recuperar secuencias sedimentarias holocenas en las que se abarcan las cronologías neolíticas de ocupación del yacimiento (ca. VI milenio A.C.).

En este trabajo se presentan los resultados del estudio estratigráfico, sedimentológico y geoquímico del sondeo LAC1. Los datos obtenidos de los distintos análisis sedimentarios y geoquímicos denotan una tendencia hacia la aridificación a lo largo del Holoceno, representada por un ciclo de agradación-incisión en la evolución fluvial del río Odrón. En el registro sedimentario fluvial se detectan restos de actividad antrópica coetánea a una de las fases finales del yacimiento que, por la presencia de un foliáceo, podríamos situar en torno a finales del IV milenio A.C. (Fase III: 


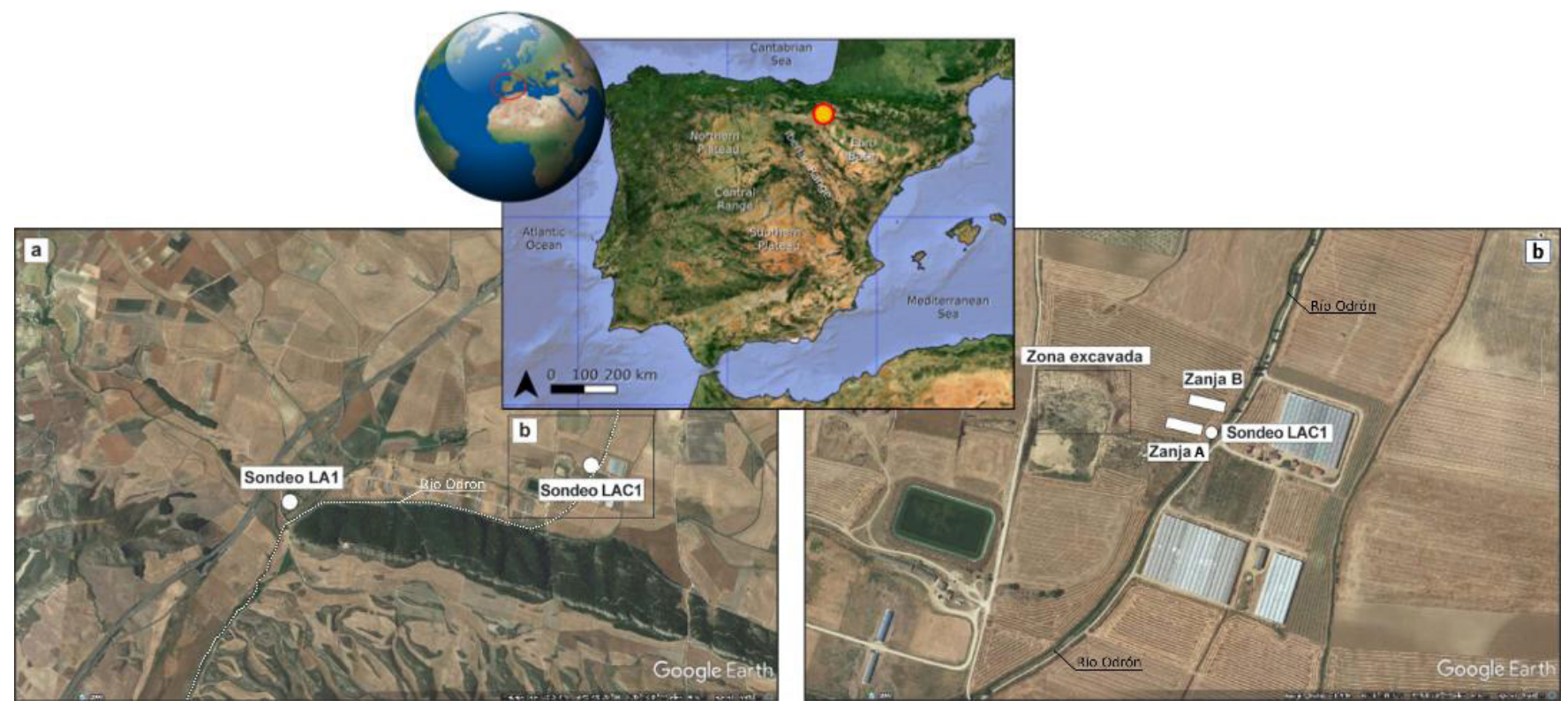

Figura 1 Localización de los sondeos y zanjas mecánicas efectuadas en las inmediaciones del poblado neolítico de Los Cascajos (Los Arcos, Navarra). Localización del sondeo LAC1: UTM 30T 42,5367 -2,1864.

Neolítico-Calcolítico). Todo ello permite situar la ocupación neolítica del yacimiento en momentos hidrológicamente más húmedos que el actual, y evidenciar la posterior aridificación de esta zona del valle del Ebro a lo largo del Holoceno reciente.

\section{Material y metodología}

\subsection{SONDEOS Y EXGAVAGIONES MEGÁNICAS}

Los sondeos mecánicos se realizaron con una perforadora mecánica por percusión portátil Van Walt/Eijkelkamp que permite la recuperación del testigo completo en maniobras de $1 \mathrm{~m}$. En el sondeo LAC1 se recuperaron $387 \mathrm{~cm}$ de registro sedimentario, llegándose al sustrato de margas miocenas. Tras la obtención de los testigos de sondeo estos fueron debidamente sellados, rotulados y guardados en una cámara frigorífica a $3-4^{\circ} \mathrm{C}$ hasta su apertura para ser analizados y muestreados.

Las zanjas, de aproximadamente $4 \times 8 \mathrm{~m}$ de extensión y $3 \mathrm{~m}$ de profundidad máxima se realiza- ron con una pala excavadora, monitorizándose en todo momento el sedimento extraído y la posible presencia de restos arqueológicos.

\subsection{ANÁLISIS GEOQUÍMICO MEDIANTE XRF-CS}

La composición elemental de los sondeos obtenidos en el yacimiento de Los Cascajos ha sido analizada mediante un escáner de fluorescencia de rayos X (XRF-CS) marca Avaatech XRF-Core Scanner. Los análisis geoquímicos se realizaron en el Laboratorio de XRF Core Scanner de la Facultad de Geología de la Universidad de Barcelona. Se trata de un método no destructivo y semicuantitativo que permite el análisis de la composición química elemental y la obtención de fotografias digitales de alta resolución (Figura 2).

La preparación de la muestra consistió en el corte y apertura longitudinal del sondeo, siendo utilizada una de las mitades para ser analizada geoquímicamente y fotografiada por la cámara de alta resolución del propio escáner. El análisis geoquímico se realizó con un intervalo de medida de 1 
cm utilizando una lámpara de Rodio y dos intensidades de medida, $10 \mathrm{kV}$ y $30 \mathrm{kV}$. La primera de las mediciones se realizó con un voltaje de $10 \mathrm{kV}$, una intensidad de corriente de $1000 \mathrm{~mA}$, y un tiempo de medida de $10 \mathrm{~s}$, habiéndose medido el contenido en los siguientes elementos: Aluminio (Al), Silicio (Si), Fósforo (P), Azufre (S), Cloro (Cl), Argón (Ar), Potasio (K), Calcio $(\mathrm{Ca})$ Titanio (Ti), Vanadio $(\mathrm{V})$, Cromo (Cr), Manganeso (Mn), Hierro (Fe), Rodio (Rh) y Plata (Ag). La segunda de las medidas se hizo con un voltaje de $30 \mathrm{kV}$, una intensidad de corriente de $2000 \mathrm{~mA}$, un tiempo de medida de 25 s y la utilización de un filtro de Paladio, habiéndose medido los siguientes elementos: Níquel (Ni), Cobre (Cu), Zinc (Zn), Galio (Ga), Germanio (Ge), Arsénico (As), Bromo (Br), Rubidio (Rb), Estroncio $(\mathrm{Sr})$, Ytrio (Y), Zirconio (Zr), Niobio (Nb), Plomo $(\mathrm{Pb})$ y Torio $(\mathrm{Th})$.

\subsection{ANÁLISIS ESTADÍSTICO DE LOS DATOS: ANÁLISIS DE GOMPONENTES PRINGIPALES}

Los datos geoquímicos obtenidos fueron filtrados y analizados utilizando métodos de estadística multivariante, en este caso el análisis de compo- nentes principales (ACP), que permitirán su mejor interpretación. El ACP es una técnica estadística de síntesis de la información, o reducción de la dimensión (número de variables) (Hotelling, 1933). Un aspecto clave en el ACP es la interpretación de los factores, ya que ésta no viene dada a priori, sino que es deducida tras observar la relación de los factores con las variables iniciales (analizándose tanto el signo como la magnitud de las correlaciones). Así, es posible reconocer e interpretar distintos procesos ambientales y/o antrópicos (reflejados en los distintos factores) que controlan/ explican la variabilidad inherente presente en los resultados analíticos. El análisis estadístico se ha realizado con el programa SPSS 20.0.

\section{Resultados}

\subsection{ESTRATIGRAFÍA Y SEDIMENTOLOGÍA}

El estudio litoestratigráfico del sondeo LAC1 y las zanjas ha permitido diferenciar 3 unidades estratigráficas en base a sus características sedimentológicas (figuras 1, 2 y 3 ):

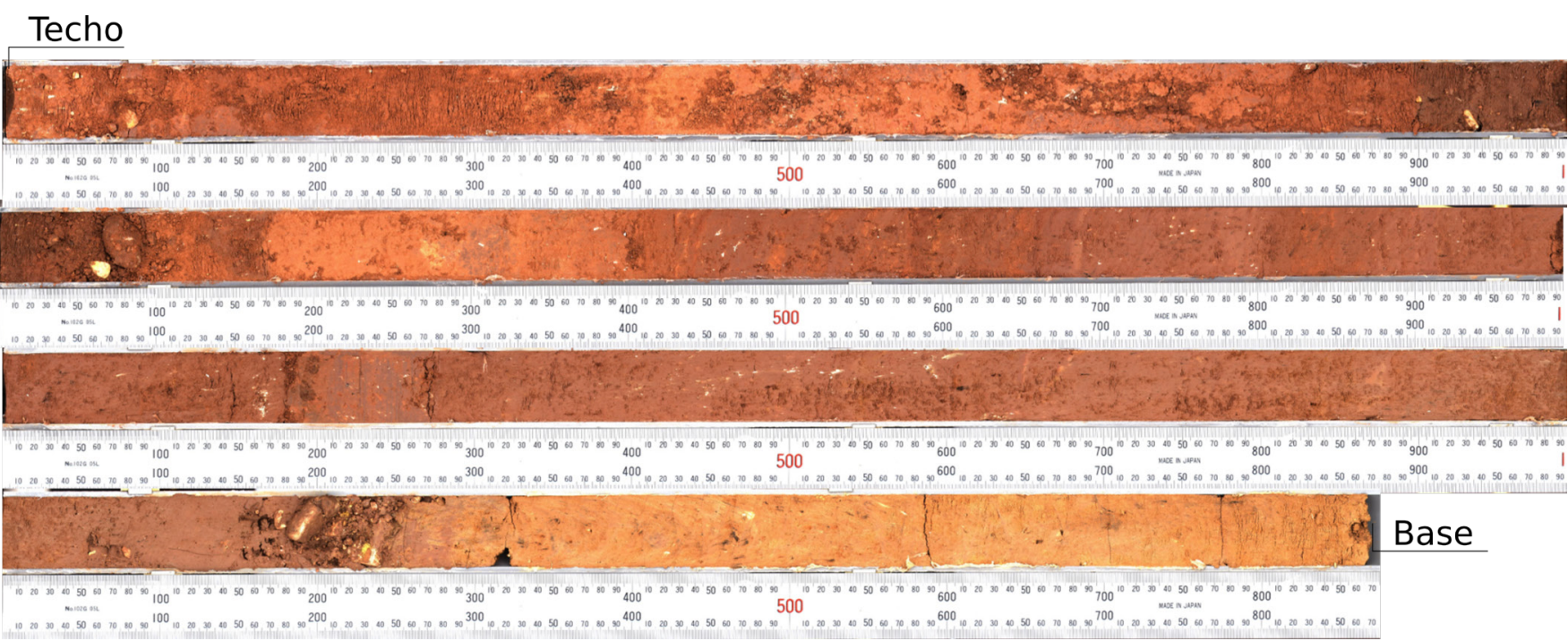

Figura 2 Testigos de sondeo recuperados en el sondeo LAC1. El techo de la secuencia está arriba a la izquierda y la base abajo a la derecha. La longitud total del sondeo es de $387 \mathrm{~cm}$. 
Unidad 1. Margas basales: Los $62 \mathrm{~cm}$ basales corresponden a margas amarillas masivas con pequeños nódulos carbonatados y fragmentos carbonosos dispersos (figuras 2 y 3e). A techo de la unidad, se presenta un contacto neto y erosivo con las gravas del nivel suprayacente, pudiéndose apreciar marcas de bioturbación en la zona de contacto que denotan la presencia de un paleosuelo parcialmente erosionado.

Unidad 2. Sedimentos fluviales: Los siguientes $208 \mathrm{~cm}$ (entre 325 y $117 \mathrm{~cm}$ de profundidad) están caracterizados por la presencia de arcillas rojas masivas de tonalidades oscuras (figuras 2, $3 \mathrm{~d}$ y $3 \mathrm{e}$ ). A base se observa un nivel de $10 \mathrm{~cm}$ de grosor compuesto por gravas de matriz arenosa. Las arcillas engloban, sobre todo en la mitad superior, intervalos ricos en precipitados blanquecinos de yeso. De la misma manera, entre los 150 y los $215 \mathrm{~cm}$ de profundidad destaca un intervalo con abundantes fragmentos de carbón. A esta altura estratigráfica se detectaron en la Zanja A dos hogares intactos con restos líticos típicos del Neolítico Final-Calcolítico (Figura 3f) en particular una punta de flecha foliácea fragmentada con retoque plano, característica de este horizonte regional en monumentos megalíticos como Longar o Aizibita (Laborda Martínez, 2016). A techo el sedimento es más limo-arenoso y compacto y su contacto con la unidad suprayacente es neto y erosivo.

Unidad 3. Sedimentos coluviales: Entre el techo de la secuencia y $117 \mathrm{~cm}$ de profundidad se extienden limos arenosos de color más amarillento y aspecto compacto y menos plástico que las arcillas subyacentes (figuras 2 y $3 d$ ). Incorpora gravas dispersas de tamaño centimétrico. Incluye en la base (entre 90 y $110 \mathrm{~cm}$ de profundidad) un nivel semejante a las arcillas rojas inferiores, con fragmentos de carbón. Presenta niveles con precipitados de yeso, si bien éste está presente de manera dispersa a lo largo de todo su espesor, excepto en el intervalo basal previamente indicado.

Aunque se trató de datar mediante radiocarbono 2 muestras procedentes de la base y el techo de la
Unidad 2, las dataciones no dieron resultado debido a la baja concentración de materia orgánica en las muestras.

\subsection{DATOS GEOQUÍMICOS Y TRATAMIENTO ESTADÍSTICO}

El análisis geoquímico y el tratamiento estadístico mediante ACP de los datos obtenidos, permiten corroborar la estratigrafía antes descrita e inferir distintos procesos sedimentarios reflejados en los componentes principales diferenciados (Figura 4). El ACP realizado explica el $79.7 \%$ de la varianza de los datos geoquímicos obtenidos. Se han extraído 4 componentes principales controlados por distintos elementos como puede observarse en la matriz factorial (Figura 4).

El primer componente principal (PG1) explica el $34.7 \%$ de la variabilidad de los datos geoquímicos. Este componente tiene una correlación positiva muy alta con los elementos Al, Ca, Si, Ti, y K (superior a 0.8 ) y en menor medida, pero con valores significativos (ca. 0.7) con el Sr y Fe (Figura 4). Este componente parece asociarse a la sedimentación fluvial terrígena de arcillas siliciclásticas (aluminosilicatos) y carbonatadas provenientes de la erosión del sustrato rocoso cenozoico aguas arriba.

El PC2 explica el 18.5\% de la variabilidad de los datos geoquímicos. Presenta una muy alta correlación positiva con el Zn (0.86) y una correlación alta con el Rb y el Fe ( $c a$. 0.7). Tiene una correlación negativa alta con el Zr (-0.75) (Figura 4). Este componente estaría mostrando variaciones en la granulometría de los sedimentos, indicando la presencia de arenas relativamente enriquecidas en minerales pesados como el zirconio. A su vez, y dada la correlación positiva obtenida con metales biófilos como el Zn, este PG2 también podría estar señalando la existencia de granulometrías finas, arcillas con cierto contenido orgánico de origen, probablemente, edáfico.

El PC3 explica el 17.1\% de la variabilidad de los datos geoquímicos. Presenta una muy alta corre- 


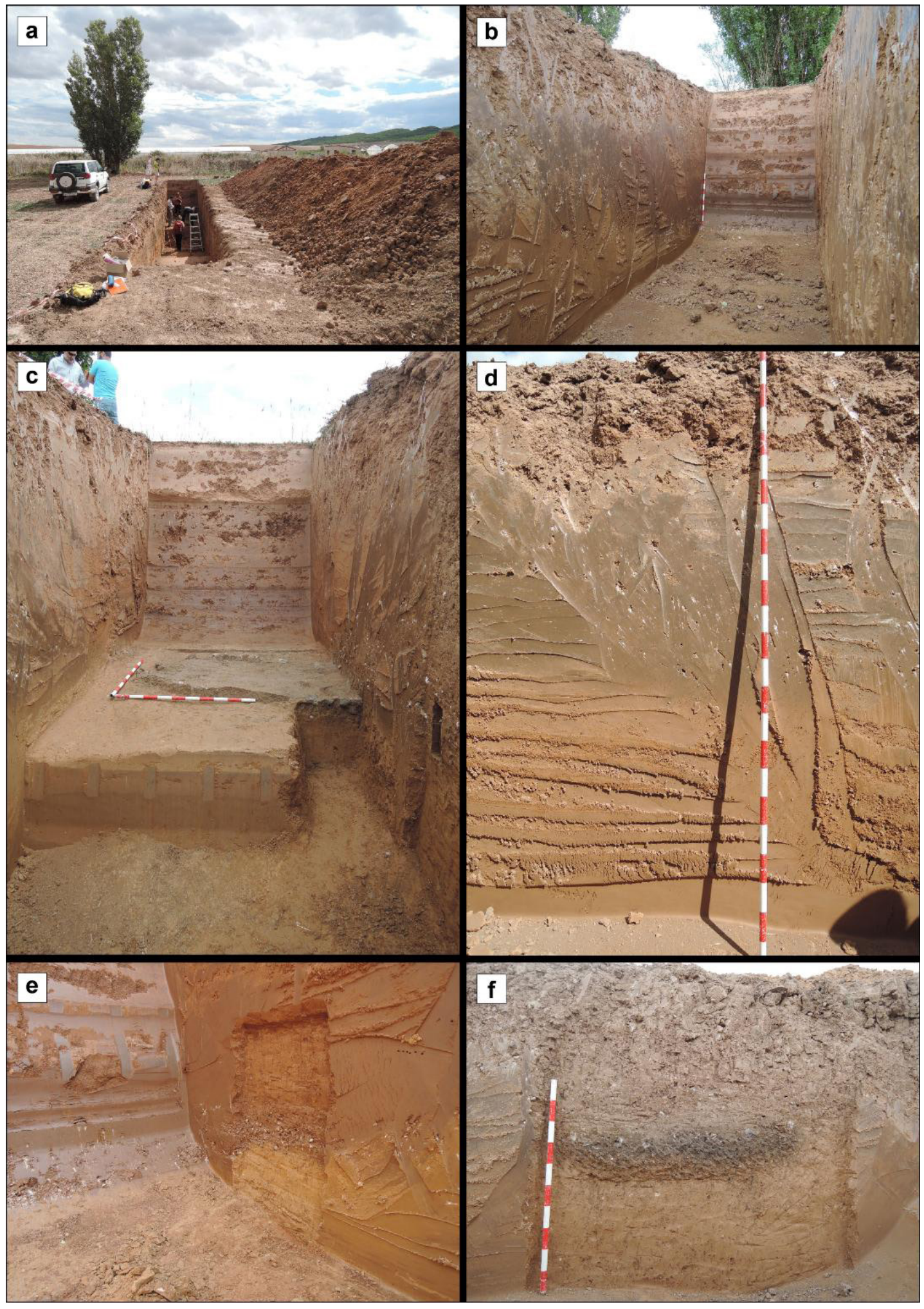

Figura 3 A: Aspecto general de la Zanja A. B: Aspecto general de la Zanja B. C: Hoguera delimitada por clastos excavada en la Zanja A (Unidad 2). D: Contacto entre la Unidad 2 (abajo, naranja) y la Unidad 3 (arriba, verdosa con clastos blancos) en la Zanja A. E: Contacto entre la Unidad 1(abajo, amarilla) y la Unidad 2 (arriba, roja) en la Zanja B. F: Otra hoguera en el perfil occidental de la Zanja A a techo de la Unidad 2. 
lación positiva con el $\mathrm{P}$ y el $\mathrm{Cl}(<0.8)$ y una muy alta correlación negativa con el S (-0.91) (Figura 4). Estos datos estarían reflejando la precipitación del yeso $\left(\mathrm{CaSO}_{4}-2 \mathrm{H}_{2} \mathrm{O}\right)$ en detrimento de la materia orgánica en los horizontes edáficos más superficiales (p. ej. P).

El PC4 explica el 9.4\% de la variabilidad y presenta una muy alta correlación positiva con el Y (0.86) y una alta correlación con el Mn (0.59). La presencia de $\mathrm{Y}$ podría asociarse a la presencia de carbón y/o materia orgánica vegetal proveniente de plantas comestibles o plantas leñosas, cuyas semillas son las que mayor concentración de este elemento presentan (Emsley, 2001). El Mn, seguramente en forma de óxido, se formaría debido a condiciones de reducción-oxidación variables que podrían derivar de una cíclica actividad de inundación-desecación de la llanura de inundación. Ambos elementos podrían ser testimonio de actividades antrópicas debido a la adición de carbón (hogares, etc.) y tal vez, al cultivo y arado de la tierra, o variaciones en la saturación de agua de los sedimentos debido a crecidas, como posibles responsables de la creación de condiciones variables de oxidación (formación de óxidos de Mn).

Además, se han calculado las puntuaciones factoriales de los análisis geoquímicos, es decir, las puntuaciones que tienen los componentes principales (y los procesos inferidos que los controlan) para cada análisis elemental a lo largo del sondeo, lo que permite conocer su evolución en el tiempo, fácilmente observable mediante su representación gráfica (PC1, PC2, PC3 y PC4 en Figura 5).

\section{Discusión}

A pesar del resultado negativo obtenido en la datación de distintas muestras de las secuencias observadas tanto en los perfiles de las zanjas como del sondeo LAC1, el hecho de haber recuperado restos arqueológicos de la Fase III de ocupación del Neolítico Final-Calcolítico (hogueras e industria lítica), permiten tener la certeza de haber recuperado un registro arqueosedimentario que abarca tanto el Neolítico como parte del Holoceno previo. Así, el estudio del sondeo junto con la observación de los perfiles de las zanjas permite situar la ocupación neolítica del yacimiento de Los Cascajos en su contexto paleoambiental.

Pueden definirse, sobre la base de margas miocenas (U1), dos unidades sedimentarias (U2 y U3) (figuras 2 y 3 ) que permiten inferir cómo fue la evolución sedimentaria del río Odrón en las inmediaciones del yacimiento, y el impacto antrópico de los pobladores de Los Cascajos en los sedimentos fluviales depositados en sus orillas.

\subsection{INGISIÓN Y AGRADAGIÓN FLUVIAL DEL RÍO ODRÓN}

La secuencia sedimentaria estudiada presenta un claro carácter fluvial. Muestra una fase de agrada-
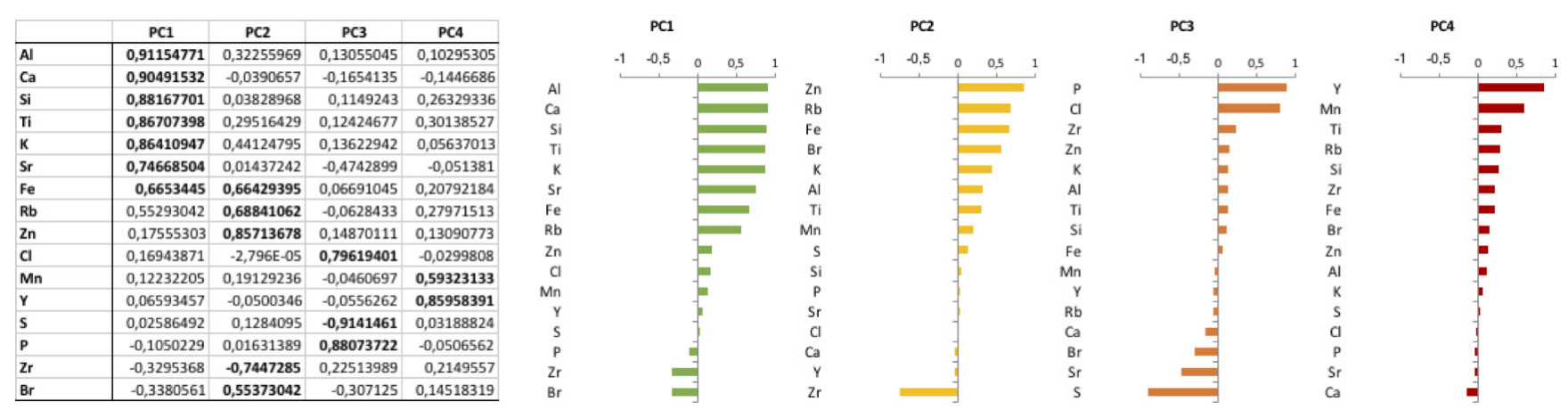

Figura 4 Matriz factorial y representación gráfica de los coeficientes factoriales de cada elemento químico analizado en los distintos componentes principales obtenidos en el ACP. 
ción fluvial que rellenó de sedimento el valle del río Odrón desde inicios del Holoceno (U2) hasta probablemente el Holoceno medio (U3) (Figura 5). Dicha etapa de agradación está flanqueada por etapas de incisión fluvial, tanto previa, durante el final del Pleistoceno, como posteriormente en el Holoceno más reciente, seguramente durante los últimos ca. 4000 años. Ambas etapas de incisión fluvial reflejan intervalos de condiciones relativamente más áridas, de menor precipitación y actividad fluvial que ya han sido registradas en secuencias fluvio-lacustres a lo largo del valle del Ebro (Sancho et al., 2008; González-Sampériz et al., 2008, 2017; González-Amuchástegui y Serrano, 2015; Aranbarri et al., 2016; Luzón et al., 2017; Morellón et al., 2018). Estas condiciones más secas, de menor escorrentía superficial y caudal fluvial, formarían un sistema fluvial de relativamente escaso caudal y energía, sin apenas capacidad de erosión y transporte sedimentario, limitándose a desarrollar fases de encajamiento del valle análogas al funcionamiento actual del río Odrón.

Sin embargo, a inicios de la Unidad 2 la secuencia de agradación de sedimentos fluviales, indicaría un curso fluvial de mayor caudal, abundantes eventos de crecidas/inundación y gran capacidad de transporte sedimentario. Todo ello requeriría de condiciones más húmedas, una mayor pluviometría, y por tanto de escorrentía superficial, que darían lugar a condiciones de mayor erosión en las laderas, es decir, mayor disponibilidad sedimentaria, y un curso fluvial capaz de redistribuir dichos sedimentos, rellenando el valle del río Odrón, que entonces discurriría a casi $4 \mathrm{~m}$ por debajo de la cota del terreno actual en el lugar del sondeo LAC1. Esta fase incluiría el inicio del Holoceno y el denominado Optimo Climático Holoceno (aprox.11600 a 5000 años cal AC), caracterizado por un aumento significativo de la humedad y que pasaría a condiciones cada vez más áridas a partir del Holoceno medio (Sancho et al., 2008; González-Amuchástegui y Serrano, 2015; Aranbarri et al., 2016; González-Sampériz et al., 2017; Luzón et al., 2017; Morellón et al., 2018).

\subsection{AMBIENTES Y EVOLUGIÓN SEDIMENTARIA FLUVIAL EN EL HOLOGENO INIGIAL}

El carácter y las variaciones de las facies sedimentarias observadas en cada unidad litoestratigráfica, permiten inferir las características del sistema fluvial a lo largo de la sección holocena recuperada, así como los principales cambios en su evolución. Durante la Unidad 2, aproximadamente la primera mitad del Holoceno, la constatación de facies de canal lateralmente equivalentes a limos orgánicos de llanura de inundación, indica la presencia de un sistema fluvial de energía media/baja y perfil longitudinal de pendiente relativamente baja. El pequeño espesor de las gravas y arenas de la secuencia de relleno de canal indica la presencia de canales relativamente poco profundos ( $c a$. de 30-50 cm), anchuras inferiores a la decena de metros y energía moderada, dado que las gravas centimétricas no son abundantes. Dichos canales podrían ser de geometrías meandriformes, con canales que migrarían lateralmente en un fondo de valle vegetado, rellenos de secuencias de canal amalgamadas, que denotan eventos de crecidas moderadas, y flanqueados por ambientes de llanura de inundación estables en el tiempo. En las arcillas de llanura de inundación es posible acotar distintos eventos de inundación gracias al reconocimiento de hasta 6 ciclos de paleosuelos agradantes (P1 a P6 en Figura 5). Así, una inundación supondría la adición de sedimento arcilloso (PC1), caracterizados por una posterior incorporación de material orgánico, posiblemente debido a la edafización y colonización por cobertera vegetal (PC2) del área de llanura de inundación. Posteriormente, se detecta la precipitación de yeso (S) en la porosidad de la zona superficial de cada evento de inundación o paleosuperficie, debido a la evaporación del agua intersticial al quedar expuesto subaéreamente (PC3). Cabe señalar la presencia dentro de la Unidad 2, entre $150 \mathrm{~cm}$ y $217 \mathrm{~cm}$ de profundidad, de un intervalo relativamente rico en $\mathrm{Mn}$ e Y (PC4) que podría indicar la mayor presencia de carbón disperso y alteración antrópica de los se- 


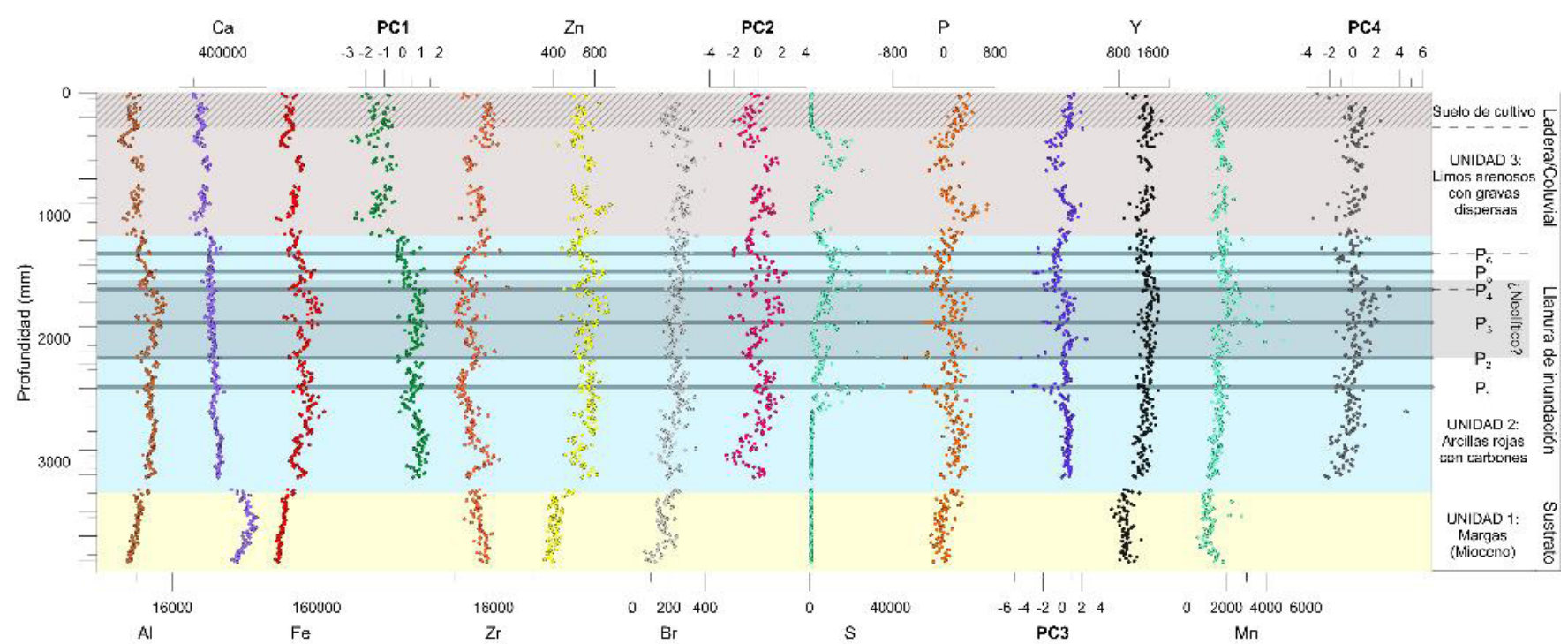

Figura 5 Representación de la evolución a lo largo del sondeo LAC1 del contenido en los elementos químicos más significativos y evolución vertical de las puntuaciones factoriales de los distintos componentes principales (PC1, PC2, PC3 y PC4). Se señalan las distintas unidades litoestratigráficas diferenciadas y su interpretación, así como los niveles de paleosuelos más significativos identificados en la Unidad 2 (P1 a P6).

dimentos (¿arado y cultivo?); este intervalo coincide con la aparición de abundantes fragmentos de carbón disperso en el sedimento en una posición estratigráfica correlacionable a la de las hogueras encontradas en la Zanja A y que potencialmente representarían el lapso cronológico de la Fase III (Neolítico Final-Calcolítico) de ocupación del yacimiento.

Las características sedimentológicas de la Unidad 3 indican un cambio en el carácter del sistema fluvial a partir de probablemente el Holoceno medio (ca. III milenio AG). Tras un evento de hídrico importante, marcado por un nivel basal de relleno de canal de gravas arenosas que podría corresponder a la formación de canales erosivos de escorrentía superficial en la ladera, se depositan limos y arcillas menos carbonatadas $(\mathrm{Ca})$ y más arenosas $(\mathrm{Zr}$, PC2) (Figura 5), con abundantes cantos de gravas fluviales de gran tamaño derivados de la erosión de la terraza pleistocena cercana ladera arriba, y una precipitación/resedimentación casi continua de yeso (S) (Figura 5). Todo ello denota un cambio en el aporte y tipo de sedimento, dándose menores tasas de sedimentación y depósitos provenientes ahora mayoritariamente de la erosión de la parte alta de la ladera donde afloran las arcillas, arenas y yesos terciarios, así como de la terraza pleistocena sobre la que se instauró el poblado neolítico. Es significativa la presencia de intervalos relativamente ricos en carbón (a $35 \mathrm{~cm}$ y $100 \mathrm{~cm}$ de profundidad) que podrían indicar la presencia de actividades antrópicas en el área en periodos posteriores al Neolítico.

Esta unidad representa el final de la fase de agradación y el inicio de la de incisión o encajamiento fluvial, lo que supuso una cada vez menor incidencia de las inundaciones en la zona estudiada. Por todo ello, la actividad antrópica en los márgenes del canal fluvial se vería facilitada, con un caudal fluvial relativamente bajo, seguramente relacionado a una creciente aridez, hacia el Holoceno Medio-Final (Sancho et al., 2008; González-Amuchástegui y Serrano, 2015; González-Sampériz et al., 2017; Luzón et al., 2017). Este hecho supondría una menor capacidad de transporte del curso fluvial, así como crecidas e inundaciones menos numerosas e intensas. Se habría iniciado por tanto la etapa de incisión fluvial que perdura hasta la 
actualidad. Todo ello haría también que el potencial de preservación de restos arqueológicos fuera inferior, debido principalmente a una menor tasa de sedimentación y mayor exposición subaérea de los restos.

Este cambio de tendencia a condiciones más áridas al final del Holoceno es el mayor cambio climático observado en el registro estudiado, y está en consonancia con numerosos registros con la misma tendencia estudiados a lo largo del área mediterránea del sur de Europa, reflejada principalmente por un descenso de taxones mesófilos (p.ej. González-Sampériz et al., 2008; 2017; Di Rita y Magri, 2009; Carrión et al., 2010; Anderson et al., 2011; Pérez-Obiol et al., 2011; Sadori et al., 2011; Jiménez-Moreno y Anderson, 2012; Magri et al., 2015; Revelles et al., 2015; 2018) y niveles lacustres más bajos (p. ej. Magny et al., 2002; Morellón et al., 2009; Corella et al., 201 1; Pellicer et al., 2016).

Finalmente, los $30 \mathrm{~cm}$ superiores se presentan alterados por el arado del terreno y el crecimiento de cereales derivados de la actividad agrícola actual.

\section{Conclusiones}

El desarrollo del poblado neolítico de Los Cascajos a orillas del río Odrón, hace de esta área y del registro sedimentario fluvial registrado en ella, una inmejorable candidata para la contextualización ambiental de dicho yacimiento. El estudio estratigráfico y sedimentológico, así como el análisis geoquímico de alta resolución, ha permitido establecer un contexto ambiental y reconstruir la evolución temporal de distintas variables paleoambientales, así como el reconocimiento de distintas actividades antrópicas desarrolladas en los márgenes del río.

El registro sedimentario estudiado recoge un ciclo de agradación fluvial que seguramente arranca a inicios del Holoceno e incluye una secuencia sedimentaria hasta el Holoceno medio post-Neolítico. En este registro queda englobada una unidad sedimentaria (Unidad 2) caracterizada por la presencia de hogares, carbones y restos arqueológicos que denotan su contemporaneidad con, al menos, la fase más reciente de ocupación (Fase III: Neolítico Final-Calcolítico) del poblado de Los Cascajos. Dicha unidad presenta características que indican la formación de una secuencia agradante de paleosuelos fluviales sobre los que los grupos humanos del poblado realizaron fuegos y tal vez actividades agrícolas. Esta dinámica sedimentaria y de uso de la llanura de inundación del río Odrón se dio en un contexto más húmedo que el actual. Esas condiciones húmedas cambiaron en una tendencia hacia un clima más árido, típico del Holoceno medio-final. Dicha aridez provocaría el descenso del nivel freático e incisión fluvial, y por tanto menor inundabilidad y agradación fluvial en el área, lo que dio lugar a la instauración de una sedimentación coluvial (Unidad 3) que también incluye periodos de actividad antrópica más tardíos.

Finalmente, no cabe duda de que, a partir de estos primeros datos, futuros trabajos de interpretación de sondeos cercanos (p. ej. LAl) y su correlación, junto con un mayor esfuerzo y aplicación de otras técnicas de datación (p. ej. OSL) y estudio (polen, análisis $\mathrm{C} / \mathrm{N}$, etc.) que completen la información de estos registros, permitirán un mayor y más preciso conocimiento de todos los procesos y cambios ambientales discutidos en este trabajo.

\section{Agradecimientos}

Los autores agradecen a la Dirección General de Cultura-Institución Príncipe de Viana del Gobierno de Navarra la subvención de las tareas de investigación realizadas a lo largo de este proyecto. Agradecemos también la labor del Dr. Ángel Carrancho (UBU, Editor Invitado); a C. Tejedor-Rodríguez e I. García-Martínez de Lagrán, investigadores del Programa "Juan de la Cierva" del Ministerio de Ciencia, Innovación y Univer- 
sidades; y las correcciones y sugerencias de la Dra. Arantxa Aranburu (EHU/UPV) y un revisor anónimo que han contribuido a mejorar el manuscrito.

\section{Referencias}

Alday, A., Domingo, R., Sebastián, M., Soto, A., Aranbarri, J., González-Sampériz, P., Sampietro-Vattuone, M.M., Utrilla, P., Montes, L., Peña-Monné, J.L., 2018, The silence of the layers: Archaeological site visibility in the Pleistocene-Holocene transition at the Ebro Basin: Quaternary Science Reviews, 184, 85-106.

Anderson, R.S., Jiménez-Moreno, G., Carrión, J.S., Pérez-Martínez, C., 2011, Postglacial history of alpine vegetation, fire, and climate from Laguna de Río Seco, Sierra Nevada, southern Spain: Quaternary Science Reviews, 30(13-14), 1615-1629.

Aranbarri, J., González-Sampériz, P., ValeroGarcés, B., Moreno, A., Gil-Romera, G., Sevilla-Callejo, M., García-Prieto, E., Di Rita, F., Mata, M.P., Morellón, M., Magri, D., Rodríguez-Lázaro, J., Carrión, J.S., 2014, Rapid climatic changes and resilient vegetation during the Lateglacial and Holocene in a continental region of southwestern Europe: Global and Planetary Change, 114, 50-65.

Aranbarri, J., Bartolomé, M., Alcolea, M., Sancho, C., Celant, A., González-Sampériz, P., Arenas, C., Magri, D., Rodríguez-Lázaro, J., 2016, Palaeobotanical insights from EarlyMid Holocene fluvial tufas in the Moncayo Natural Park (Iberian Range, NE Spain): Regional correlations and biogeographic implications: Review of Palaeobotany and Palynology, 234, 31-43.

Carrión, J.S., Fernández, S., González-Sampériz, P., Gil-Romera, G., Badal, E., CarriónMarco, Y., López-Merino, L., López-Sáez, J.A., Fierro, E., Burjachs, F., 2010, Expected trends and surprises in the Lateglacial and Holocene vegetation history of the Iberian Peninsula and Balearic Islands: Review of Palaeobotany and Palynology, 162(3), 458-475.

Corella, J., Moreno, A., Morellón, M., Rull, V., Giralt, S., Rico, M., Pérez-Sanz, A., ValeroGarcés, B., 2011, Climate and human impact on a meromictic lake during the last 6,000 years (Montcortès Lake, Central Pyrenees, Spain): Journal of Paleolimnology, 46(3), 351-367.

Di Rita, F., Magri, D., 2009, Holocene drought, deforestation and evergreen vegetation development in the central Mediterranean: a 5500 year record from Lago Alimini Piccolo, Apulia, southeast Italy: The Holocene 19(2), 295-306.

Emsley,J., 2001, Nature's Building Blocks: An A-Z Guide to the Elements: Oxford, RU, Oxford University Press, 538.

García-Gazólaz, J., Sesma-Sesma, J., 2001, Los Cascajos (Los Arcos, Navarra). Intervenciones 1996-1999: Trabajos de Arqueología Navarra, 15, 299-306.

García-Gazólaz, J., Sesma-Sesma, J., 2005, Dispositivos de combustión durante la Prehistoria reciente de Navarra: Munibe (Antropologia-Arkeologia), 57, 259-273.

García-Martínez de Lagrán, I., Iriarte, E., GarcíaGazólaz, J., Tejedor-Rodríguez, C., GibajaBao, J.F., Moreno-García, M., Pérez-Jordá, G., Ruiz-Alonso, M., Sesma-Sesma, J., Garrido-Pena, R., Carrancho-Alonso, A., Peña-Chocarro, L., Rojo-Guerra, M.A., 2016, 8.2 ka BP paleoclimatic event and the Ebro Valley Mesolithic groups: Preliminary data from Artusia rock shelter (Unzué, Navarra, Spain): Quaternary International, 403, 151-173.

González-Amuchastegui, M.J., Serrano, E., 2015, Tufa buildups, landscape evolution and human impact during the Holocene in the Upper Ebro Basin: Quaternary International, 364, 54-64. 
González-Sampériz, P., Valero-Garcés, B.L., Moreno, A., Morellón, M., Navas, A., Machín, J., Delgado-Huertas, A., 2008, Vegetation changes and hydrological fluctuations in the Central Ebro Basin (NE Spain) since the Late Glacial period: Saline lake records: Palaeogeography, Palaeoclimatology, Palaeoecology, 259(2-3), 157-181.

González-Sampériz, P., Aranbarri, J., PérezSanz, A., Gil-Romera, G., Moreno, A., Leunda, M., Sevilla-Callejo, M., Corella, J.P., Morellón, M., Oliva, B., Valero-Garcés, B., 2017, Environmental and climate change in the southern Central Pyrenees since the Last Glacial Maximum: A view from the lake records: CATENA, 149(3), 668-688.

Hotelling, H., 1933, Analysis of a complex of statistical variables into principal components:. Journal of Educational Psychology, 24(6), 417-441.

Jiménez-Moreno, G., Anderson, R.S., 2012, Holocene vegetation and climate change re- corded in alpine bog sediments from the Borreguiles de la Virgen, Sierra Nevada, southern Spain: Quaternary Research, 77(1), 44-53.

Laborda-Martínez, M.A., 2016, Análisis funcional de las puntas de flecha de los contextos sepulcrales de los monumentos megalíticos de Aizbita, Charracadía y Morea (valle del Salado, Navarra): Trabajos de Arqueología Navarra, 28, 115-156.

Luzón, A., Gauthier, A., Pérez,A.,Pueyo-Anchuela, O., Mayayo, M.J., Muñoz, A., 2017, LatePleistocene-Holocene palaeoenvironmental evolution of the Añamaza River valley (Iberian Range, NE Spain): Multidisciplinary approach on the study of carbonate fluvial systems: Quaternary International, 437(A), $51-70$.

Magny, M., Miramont, C., Sivan, O., 2002, Assessment of the impact of climate and anthropogenic factors on Holocene
Mediterranean vegetation in Europe on the basis of palaeohydrological records: Palaeogeography, Palaeoclimatology, Palaeoecology, 186(1-2), 47-59.

Magri, D., Agrillo, E., Di Rita, F., Furlanetto, G., Pini, R., Ravazzi, C., Spada, F., 2015, Holocene dynamics of tree taxa populations in Italy: Review of Palaeobotany and Palynology, 218, 267-284.

Morellón, M., Valero-Garcés, B., VegasVilarrúbia, T., González-Sampériz, P., Romero, Ó., Delgado-Huertas, A., Mata, P., Moreno, A., Rico, M., Corella, J.P., 2009, Lateglacial and Holocene palaeohydrology in the western Mediterranean region: the Lake Estanya record (NE Spain): Quaternary Science Reviews, 28(25-26), 2582-2599.

Morellón, M., Aranbarri,J., Moreno, A., GonzálezSampériz, P., Valero-Garcés, B., 2018. Early Holocene humidity patterns in the Iberian Peninsula reconstructed from lake, pollen and speleothem records: Quaternary Science Reviews, 181, 1-18.

Pellicer, X. M., Corella, J.P., Gutiérrez, F., Roque, C., Linares, R., Carbonel, D., Zarroca, M., Guerrero, J., Comas, X., 2016, Sedimentological and paleohydrological characterization of Late Pleistocene and Holocene tufa mound paleolakes using trenching methods in the Spanish Pyrenees: Sedimentology, 63(6), 1786-1819.

Peña-Chocarro, L., Zapata-Peña, L., GarcíaGazólaz, J., González-Morales, M., SesmaSesma, J., Straus, L.G., 2005, The spread of agriculture in northern Iberia: new archaeobotanical data from El Mirón cave (Cantabria) and the open-air site of Los Cascajos (Navarra): Vegetation History and Archaeobotany, 14(4), 268-278.

Pérez-Obiol, R., Jalut, G., Julià, R., Pèlachs, A., Iriarte-Chiapusso, M.J., Otto, T., HernándezBeloqui, B., 2011, Mid-Holocene vegetation and climatic history of the Iberian Peninsula: The Holocene, 21(1), 75-93. 
Revelles, J., Cho, S., Iriarte, E., Burjachs, F., van Geel, B., Palomo, A., Piqué, R., PeñaChocarro, L., Terradas, X., 2015, MidHolocene vegetation history and Neolithic land-use in the Lake Banyoles area (Girona, Spain): Palaeogeography, Palaeoclimatology, Palaeoecology, 435, 70-85.

Revelles, J., Burjachs, F., Palomo, A., Piqué, R., Iriarte, E., Pérez-Obiol, R., Terradas, X., 2018, Human-environment interaction during the Mesolithic-Neolithic transition in the NE Iberian Peninsula. Vegetation history, climate change and human impact during the Early-Middle Holocene in the Eastern Pre-Pyrenees: Quaternary Science Reviews, 184, 183-200.

Sadori, L., Jahns, S., Peyron, O., 2011, MidHolocene vegetation history of the central Mediterranean: The Holocene, 21(1), 117-129.

Sancho, C., Peña, J.L., Muñoz, A., Benito, G., McDonald, E., Rhodes, E.J., Longares, L.A., 2008, Holocene alluvial morphopedosedimentary record and environmental changes in the Bardenas Reales Natural Park (NE Spain): CATENA, 73, 225-238. 